\title{
Astronomy development in Thailand: the role of NARIT
}

\section{Boonrucksar Soonthornthum, Busaba Kramer* and Saran Poshyachinda}

National Astronomical Research Institute of Thailand, Physics Building, Chiang Mai University, Chang Mai 50200 Thailand

*email: busaba@narit.or.th

\begin{abstract}
Astronomy development in Thailand has improved significantly during the last few years. The government has approved the establishment of the National Astronomical Research Institute of Thailand (NARIT). Roles of NARIT in the development astronomical research and astronomy education in Thailand includes a national framework, national facilities, collaborative research networks, teacher training and public outreach programmes. The new 2.4-metre reflecting telescope will serve not only astronomy community in Thailand but also in Southeast Asia.
\end{abstract}

Keywords. Astronomy education, Thailand, astronomy development, developing country, National Observatory

\section{Background}

In 2004, the Royal Thai government approved the establishment of the National Astronomical Research Institute of Thailand (NARIT), under the Ministry of Science and Technology. NARIT is a research-emphasis institute providing a collaborative network for developing and strengthening knowledge in astronomy, so as to meet international standards. The institute also promotes education and learning culture in astronomy for the public and thus encourage them to seek further involvement with science and technology. The review of astronomy in Thailand from past to present and the roles of NARIT in both astronomical research and education in astronomy are presented in Kramer's paper (in these proceedings). In this paper we report the recent development of the Thai National Observatory.

\section{Thai National Observatory}

The main facility of NARIT is the National Observatory which is located on the very top of the highest mountain in Thailand at the altitude of 2550 metres above mean sea level. It is named Doi Intanon, in Chiang Mai province of northern Thailand, which is also renowned for the superb climate and tourist attractions. Seeing tests and weather monitoring at the site have been carried out since March 2006. The average seeing is 0.7 arc sec (Poshyachinda, private communication).

The National Observatory will operate a 2.4-metre reflecting telescope with an altazimuth drive system (see Figure 1). This system has been synchronized with automatic motion of the dome structure, so that observations can be carried out efficiently. The National Observatory and the 2.4-metre reflecting telescope are currently under construction and are expected to be operational in the beginning of the year 2009. The time-line of the development and list of astronomical instruments are presented in Table 1. 
Table 1. Time-line and list of astronomical instruments of the Thai National Observatory

\begin{tabular}{lcc}
\hline Year & Activities & Astronomical instruments \\
\hline March 2006 - & Seeing tests & \\
July 2006 - & Construction of enclosure and telescope & \\
October 2008 & Installation of telescope & \\
January 2009 & First light & $4 \mathrm{k} \times 4 \mathrm{k}$ CCD camera \\
2010 & Full operation & High resolution échelle spectrograph \\
& & \\
\hline
\end{tabular}

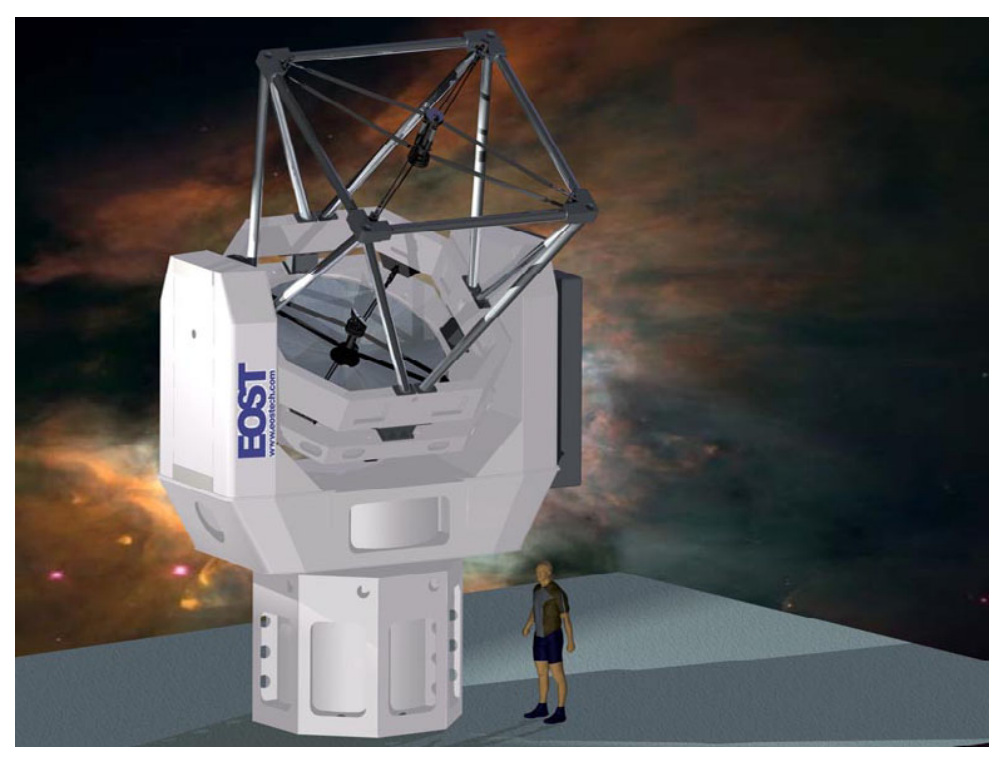

Figure 1. The 2.4-metre telescope which will be installed at the National Observatory

\section{Summary}

NARIT plays an important role in the development of astronomy in Thailand. Its aims are not only to establish the national facility but also to develop astronomical research and education in astronomy in Thailand on the national level, from schools to advanced research. The national facility will be equipped with modern astronomical instruments and will serve not only the astronomy community in Thailand but also in Southeast Asia. 